\title{
Refined carbohydrate intake in relation to non-verbal intelligence among Tehrani schoolchildren
}

\author{
Amin Salehi Abargouei ${ }^{1,2}$, Naser Kalantari ${ }^{3}$, Nasrin Omidvar ${ }^{3}$, Bahram Rashidkhani ${ }^{3}$, \\ Anahita Houshiar Rad $^{4}$, Azizeh Afkham Ebrahimi ${ }^{5}$, Hossein Khosravi-Boroujeni ${ }^{1}$ and \\ Ahmad Esmaillzadeh ${ }^{1,2, *}$ \\ ${ }^{1}$ Food Security Research Center, Isfahan University of Medical Sciences, Isfahan, Islamic Republic of Iran: \\ ${ }^{2}$ Department of Community Nutrition, School of Nutrition and Food Science, Isfahan University of Medical \\ Sciences, PO Box 81745, Isfahan, Islamic Republic of Iran: ${ }^{3}$ Department of Community Nutrition, Faculty of \\ Nutrition and Food Technology, Shahid Beheshti University of Medical Sciences, Tehran, Islamic Republic of Iran: \\ ${ }^{4}$ National Nutrition and Food Technology Institute, Shahid Beheshti University of Medical Sciences, Tehran, \\ Islamic Republic of Iran: ${ }^{5}$ Mental Health Research Center, Tehran University of Medical Sciences, Tehran, Islamic \\ Republic of Iran
}

Submitted 7 May 2011: Accepted 9 November 2011: First published online 9 December 2011

\begin{abstract}
Objective: Nutrition has long been considered one of the most important environmental factors affecting human intelligence. Although carbohydrates are the most widely studied nutrient for their possible effects on cognition, limited data are available linking usual refined carbohydrate intake and intelligence. The present study was conducted to examine the relationship between long-term refined carbohydrate intake and non-verbal intelligence among schoolchildren.

Design: Cross-sectional study.

Setting: Tehran, Iran.

Subjects: In this cross-sectional study, 245 students aged 6-7 years were selected from 129 elementary schools in two western regions of Tehran. Anthropometric measurements were carried out. Non-verbal intelligence and refined carbohydrate consumption were determined using Raven's Standard Progressive Matrices test and a modified sixty-seven-item FFQ, respectively. Data about potential confounding variables were collected. Linear regression analysis was applied to examine the relationship between non-verbal intelligence scores and refined carbohydrate consumption.

Results: Individuals in top tertile of refined carbohydrate intake had lower mean non-verbal intelligence scores in the crude model $(P<0 \cdot 038)$. This association remained significant after controlling for age, gender, birth date, birth order and breast-feeding pattern $(P=0 \cdot 045)$. However, further adjustments for mother's age, mother's education, father's education, parental occupation and BMI made the association statistically non-significant. We found a significant inverse association between refined carbohydrate consumption and non-verbal intelligence scores in regression models $(\beta=-11 \cdot 359, P<0 \cdot 001)$. This relationship remained significant in multivariate analysis after controlling for potential confounders $(\beta=-8 \cdot 495, P=0 \cdot 038)$.

Conclusions: The study provides evidence indicating an inverse relationship between refined carbohydrate consumption and non-verbal intelligence among Tehrani children aged 6-7 years. Prospective studies are needed to confirm our findings.
\end{abstract}

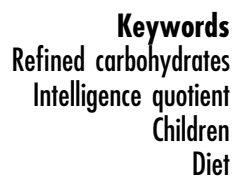

Nutrition has long been considered one of the most important environmental factors affecting human intelligence. Several studies have been performed in an effort to discern the relationship between nutrition and intelligence or cognitive ability, with the majority focusing on childhood as an important part of the life cycle.
Some investigations have assessed malnutrition in relation to cognitive ability ${ }^{(1,2)}$ and demonstrated that nutritional supplementation can improve children's cognitive ability, at least among those with low dietary intakes ${ }^{(2-4)}$. Other studies have indicated that deficiencies of $\mathrm{Fe}$, iodine, $\mathrm{Cu}$ and folic acid are associated with lower intelligence 
status $^{(5-8)}$ and that children with low dietary intakes of these micronutrients would benefit from supplementation $^{(9,10)}$. In addition to considering nutrients, dietary patterns have also been found to be associated with intelligence among children. Theodore et al. concluded that consuming fish, breads and cereals would probably improve children's cognitive development. In contrast, daily margarine consumption was inversely associated with cognitive functioning ${ }^{(11)}$.

Among macronutrients, carbohydrates are the most widely studied nutrient for their possible effects on cognition. Since glucose is the primary source of energy for the brain, several studies have explored the short-term effect of carbohydrates on cognitive ability with inconsistent results ${ }^{(12-15)}$. The most consistent findings from macronutrient manipulation and meal-based studies are related to the effects of glucose on short-term and delayed verbal and non-verbal memory performance ${ }^{(16)}$. Carbohydrate-cognitive function relationships have also been assessed by considering the glycaemic index and glycaemic load of carbohydrates. A recent review has concluded that the evidence on the relationship between dietary glycaemic load and short-term cognitive performance is not sufficient to support the association ${ }^{(15)}$. Therefore, further studies on the association between dietary glycaemic index and glycaemic load in relation to cognitive function are warranted.

The Iranian diet contains high amounts of carbohydrate from processed grains. High consumption of processed grains has been related to greater risk of obesity ${ }^{(17)}$, type 2 diabetes ${ }^{(18,19)}, \mathrm{CVD}^{(19)}$, metabolic syndrome $\mathrm{e}^{(20)}$ and high blood pressure ${ }^{(21,22)}$ even among Iranians ${ }^{(23)}$. Limited data are available linking usual carbohydrate intake from processed grains and cognitive performance. An experimental study found that a 'high fat/refined carbohydrate' diet can increase the susceptibility to spatial learning deficit in rats compared with a 'low fat/complex carbohydrate' diet ${ }^{(24)}$. We are aware of just one report that has been done in man. In a recent British cohort, the investigators indicated that adherence to a 'high fat-high sugar' dietary pattern in early childhood would unfavourably affect intelligence at 8 years of age ${ }^{(25)}$. It must be kept in mind that, in the mentioned study, dietary patterns (instead of nutrients or foods) were regarded as an exposure. In the holistic approach of dietary pattern analysis $^{(26)}$, it is impossible to identify the association of specific foods or nutrients (like carbohydrates) with cognitive function. Therefore, it would be interesting to further explore carbohydrate-intelligence quotient (IQ) relationships. Furthermore, due to high consumption of dietary carbohydrates from processed grains, the Iranian diet provides an excellent opportunity to assess such relationships within the Iranian population. The present study was therefore conducted to examine the relationship between long-term refined carbohydrate intake and IQ among schoolchildren in Tehran, Iran.

\section{Participants and methods}

\section{Study population}

The current study is a secondary analysis of data from a case-control study aiming to find the relationship between breast-feeding and myopia. In total, 245 students aged 6-7 years were selected from 129 elementary schools in two western regions of Tehran. The inclusion criteria were being 6-7 years old, not following any specific diet and no food avoidance. If the participants had any eye, metabolic or congenital disease they were excluded from the study.

\section{Antbropometric measurements}

Weight was measured with the participant wearing light clothes using a digital scale (model 803; Seca, Hamburg, Germany) to the nearest $0 \cdot 1 \mathrm{~kg}$. Each participant was weighed three times and the mean of these three measurements was considered as the final recorded weight for the participant. The participant's height was measured in standing position by the use of a stiff plastic tape measure fixed on a wall and recorded to the nearest $1 \mathrm{~cm}$. To measure height, the participant removed his/her shoes and stood with his/her head, shoulder blades, buttocks and heels touching the wall where the tape was fixed.

\section{Assessment of refined carbobydrate intake}

Dietary intake of refined carbohydrates in the last year was assessed by a modified sixty-seven-item FFQ. To construct this FFQ, we selected eighty-four food items with refined carbohydrate content that had high glycaemic index and were more frequently consumed among children and adolescents. These items consisted of rice, different types of breads, cooked potato, chips, vermicelli or macaroni, white flour and all food items produced from white flour (like biscuits, cakes and crackers), sugar and food items containing high amounts of sugar (like jam, honey, jelly, chocolates and sugarsweetened beverages). These items were selected from among a list of 168 food items included in a validated FFQ for the Iranian population ${ }^{(27)}$. We selected the food items that were more frequently used among elementaryschool children ${ }^{(28)}$. To assess the performance of the eighty-four-item FFQ, we asked forty children (twenty male and twenty female) aged 6-7 years and their parents to fill out the questionnaire. At this time a $5 \mathrm{~d}$ food diary was also requested from participants and their parents about foods consumed in these $5 \mathrm{~d}$. This was done to make sure that all food items containing refined carbohydrates had been included in the FFQ. Comparison of the eighty-four-item FFQ with the food diaries made us modify the FFQ to include sixty-seven food items more frequently consumed. The FFQ was self-administered and parents in collaboration with their children reported the frequency of each food item as well as the portion size 
that the child ate at each time. The reliability of this FFQ for assessing dietary intakes of refined carbohydrates was shown to be reasonable $(r=0 \cdot 633 ; P<0 \cdot 01)$. Consumption of all food items was considered in grams per day and then macronutrient intakes for each participant were calculated by use of the Iranian national food composition table. Total macronutrient intake was calculated by summing up macronutrients from all foods.

\section{Measurement of intelligence}

Raven's Colorful Progressive Matrices test, which has been validated among schoolchildren in Tehran ${ }^{(29)}$, was used as a screening test of cognitive ability. These matrices are non-verbal multiple-choice measures of the reasoning which is often referred to as 'general intelligence'. Raven's Progressive Matrices measures the ability to make comparisons, to reason by analogy and to organize spatial perceptions into systematically related wholes. These matrices assess the non-verbal intelligence. The test also measures the ability to think clearly and make sense of complexity, as well as the ability to store and reproduce information. The matrices consist of thirtysix colourful puzzles designed to measure a person's ability to form perceptual relationships and to reason by analogy independent of language and formal schooling, and can be used with persons ranging from 6 years of age to adulthood. The candidate is provided with multiple choices to identify the missing segment required to complete a larger pattern in each test item. In the present study, the test was administered individually under the supervision of trained research assistants, and the raw scores were converted to standardized scores based on the age of participants for analysis ${ }^{(29)}$. This test was selected because it is a valid, rapid and simple way to measure participants' intelligence and cognition status at the age of 6-7 years. Individuals with higher scores in the test have been considered to have better intelligence than those with lower scores.

\section{Assessment of other variables}

Information about physical activity, birth date, birth weight, maternal age, parental education and occupation, birth order and child's breast-feeding pattern in infancy was assessed by a self-administered questionnaire mailed to parents. Physical activity was assessed by a questionnaire asking parents about types of routine physical activity performed by their children during the day and the time they spent daily or weekly for each activity. Physical activity was then treated as a categorical variable $(<1 \mathrm{~h} / \mathrm{d}, 1-3 \mathrm{~h} / \mathrm{d},>3 \mathrm{~h} / \mathrm{d})$. To assess the pattern of breastfeeding during infancy, parents were asked to report the duration of breast-feeding and the time of introducing complementary foods before the age of 6 months. Based on this information, children were categorized into three groups of breast-feeding pattern based on WHO recommendations ${ }^{(30)}$ : not breast-fed (did not consume breast milk from birth), predominantly breast-fed (consumed breast milk for less than 6 months or consumed foods other than breast milk before the age of 6 months) and exclusively breast-fed (consumed only breast milk up to 6 months of age). Preterm children were defined as those born earlier than week 32 of gestation. A member of the research team (A.S.A.) was responsible for communicating with parents via telephone to answer parents' questions.

\section{Statistical analysis}

Histograms and the Kolmogrov-Smirnov test were used to assess the normality of the distribution of variables. Log transformation was applied for non-normally distributed variables. Tertiles of refined carbohydrate intake were used for categorization of the participants. Comparison of continuous and categorical variables across tertiles of refined carbohydrate intake was done by the use of oneway ANOVA and the $\chi^{2}$ test, respectively. Analysis of covariance (ANCOVA) was used to determine the differences in non-verbal intelligence scores across tertiles of refined carbohydrate intake. We also used multivariate linear regression analysis to assess the relationship in different models. In the first model we controlled for age, gender, birth date, birth order and breast-feeding pattern. In the second model, we further adjusted for mother's age, mother's education, father's education and parental occupation. In the third model, we additionally controlled for BMI. All statistical analyses were done using the SPSS for Windows statistical software package version $15 \cdot 0$ (SPSS Inc., Chicago, IL, USA). $P$ values $<0.05$ were considered statistically significant.

\section{Results}

In total, 245 children aged 6-7 years participated in the study; among them, eight had missing data for refined carbohydrate consumption and one reported consumption of more than $1000 \mathrm{~g} / \mathrm{d}$. After exclusion of these individuals, 236 children $(42 \cdot 4 \%$ male) remained for the current analysis.

Daily consumption of refined carbohydrates was $285 \cdot 16$ (sD $157 \cdot 03$ ) $\mathrm{g} / \mathrm{d}$. General characteristics of the total study population and across tertiles of refined carbohydrate intake are provided in Table 1. Mean (SD) age and BMI of the participants was $6 \cdot 34$ (SD 0.48 ) years and $16 \cdot 25$ (SD $2 \cdot 85) \mathrm{kg} / \mathrm{m}^{2}$, respectively. No significant differences were found in terms of general characteristics across tertiles of refined carbohydrate intake.

The score of non-verbal intelligence ranged from 77 to 146. Table 2 indicates the mean non-verbal intelligence scores in crude and adjusted models across tertiles of refined carbohydrate intake. Individuals in the top tertile of refined carbohydrate intake had lower mean nonverbal intelligence scores in the crude model $(P<0 \cdot 038)$. The difference remained significant after controlling for 
Table 1 General characteristics of the study population according to tertile of refined carbohydrate intake: children ( $n$ 245) aged $6-7$ years, Tehran, Iran

\begin{tabular}{|c|c|c|c|c|c|c|c|c|c|}
\hline & & & \multicolumn{6}{|c|}{ Tertile of refined carbohydrate consumption } & \multirow[b]{3}{*}{$P$ value } \\
\hline & \multicolumn{2}{|c|}{ Whole population } & \multicolumn{2}{|c|}{ First $(n 79)$} & \multicolumn{2}{|c|}{ Second $(n 79)$} & \multicolumn{2}{|c|}{ Third $(n 78)$} & \\
\hline & Mean & SD & Mean & SD & Mean & SD & Mean & SD & \\
\hline Age (years) & $6 \cdot 34$ & 0.48 & $6 \cdot 38$ & 0.49 & $6 \cdot 34$ & 0.50 & $6 \cdot 29$ & $0 \cdot 46$ & 0.532 \\
\hline Gender ( $\%$ of males) & \multicolumn{2}{|c|}{$42 \cdot 4$} & \multicolumn{2}{|c|}{$29 \cdot 0$} & \multicolumn{2}{|c|}{35.0} & \multicolumn{2}{|c|}{$36 \cdot 0$} & 0.446 \\
\hline Birth weight (g) & $3202 \cdot 74$ & $514 \cdot 91$ & $3177 \cdot 70$ & $551 \cdot 37$ & $3204 \cdot 51$ & $410 \cdot 04$ & $3230 \cdot 38$ & $568 \cdot 65$ & $0 \cdot 864$ \\
\hline Mother's age (years) & $34 \cdot 08$ & 4.91 & 34.97 & $4 \cdot 78$ & $33 \cdot 78$ & $4 \cdot 84$ & $33 \cdot 39$ & $5 \cdot 05$ & $0 \cdot 134$ \\
\hline Weight (kg) & $23 \cdot 84$ & $5 \cdot 12$ & $24 \cdot 28$ & $5 \cdot 28$ & $23 \cdot 31$ & $4 \cdot 70$ & $23 \cdot 92$ & $5 \cdot 38$ & 0.484 \\
\hline BMI $\left(\mathrm{kg} / \mathrm{m}^{2}\right)$ & $16 \cdot 25$ & $2 \cdot 85$ & $16 \cdot 33$ & $2 \cdot 88$ & $16 \cdot 18$ & $2 \cdot 67$ & $16 \cdot 25$ & 3.01 & 0.945 \\
\hline \multicolumn{10}{|l|}{ Birth order (\%) } \\
\hline First child & \multicolumn{2}{|c|}{$49 \cdot 3$} & \multicolumn{2}{|c|}{$46 \cdot 8$} & \multicolumn{2}{|c|}{53.9} & \multicolumn{2}{|c|}{$47 \cdot 3$} & 0.613 \\
\hline Second child & \multicolumn{2}{|c|}{$39 \cdot 3$} & \multicolumn{2}{|c|}{$39 \cdot 2$} & \multicolumn{2}{|c|}{$34 \cdot 2$} & \multicolumn{2}{|c|}{$44 \cdot 6$} & \\
\hline Third or higher & \multicolumn{2}{|c|}{$11 \cdot 4$} & \multicolumn{2}{|c|}{$14 \cdot 0$} & \multicolumn{2}{|c|}{$11 \cdot 8$} & \multicolumn{2}{|c|}{$8 \cdot 1$} & \\
\hline \multicolumn{10}{|l|}{ Father's education (\%) } \\
\hline$\leq$ High-school diploma & \multirow{2}{*}{\multicolumn{2}{|c|}{$\begin{array}{l}53 \cdot 5 \\
46 \cdot 5\end{array}$}} & \multirow{2}{*}{\multicolumn{2}{|c|}{$\begin{array}{l}45 \cdot 5 \\
54 \cdot 5\end{array}$}} & \multirow{2}{*}{\multicolumn{2}{|c|}{$\begin{array}{l}55 \cdot 3 \\
44.7\end{array}$}} & \multirow{2}{*}{\multicolumn{2}{|c|}{$\begin{array}{l}60 \cdot 3 \\
39 \cdot 7\end{array}$}} & $0 \cdot 179$ \\
\hline$>$ High-school diploma & & & & & & & & & \\
\hline \multicolumn{10}{|l|}{ Mother's education (\%) } \\
\hline$\leq$ High-school diploma & & & & & 66 & & & & $0 \cdot 130$ \\
\hline >High-school diploma & & & & & 33 & & & & \\
\hline Time of birth (\%) & & & & & & & & & \\
\hline Preterm* ${ }^{*}$ & & & & & 6 & & & & 0.263 \\
\hline Term & & & & & 93 & & & & \\
\hline Breast-feeding patternt (\%) & & & & & & & & & \\
\hline Not breast-fed & & & & & 1 & & & & 0.759 \\
\hline Predominantly breast-fed & & & & & 59 & & & & \\
\hline Exclusively breast-fed & & & & & 39 & & & & \\
\hline Physical activity $¥$ (\%) & & & & & & & & & \\
\hline$<1 \mathrm{~h} / \mathrm{d}$ & & & & & 39 & & & & 0.965 \\
\hline $1-3 \mathrm{~h} / \mathrm{d}$ & & & & & 55 & & & & \\
\hline$>3 \mathrm{~h} / \mathrm{d}$ & & & & & 5 & & & & \\
\hline
\end{tabular}

${ }^{*}$ Preterm children were defined as those born earlier than week 32 of gestation.

tNot-breast-fed: did not consume breast milk from birth; predominantly breast-fed: consumed breast milk for less than 6 months or consumed foods other than breast milk before the age of 6 months; exclusively breast-fed: consumed only breast milk up to 6 months of age.

¥Questions about physical activity were related to the type of routine physical activity performed by children during the day and time they spent daily or weekly for each activity.

Table 2 Multivariate-adjusted means of non-verbal intelligence scores across tertile of refined carbohydrate intake: children ( $n$ 245) aged 6-7 years, Tehran, Iran

\begin{tabular}{|c|c|c|c|c|c|c|c|}
\hline & \multicolumn{6}{|c|}{ Tertile of refined carbohydrate intake } & \multirow[b]{3}{*}{$P$ value* } \\
\hline & \multicolumn{2}{|c|}{ First $(n 78)$} & \multicolumn{2}{|c|}{ Second (n73) } & \multicolumn{2}{|c|}{ Third $(n 68)$} & \\
\hline & Mean & SE & Mean & SE & Mean & SE & \\
\hline 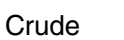 & $116 \cdot 02$ & $1 \cdot 45$ & $115 \cdot 2$ & $1 \cdot 49$ & $110 \cdot 86 \|$ & 1.55 & 0.038 \\
\hline Model 1† & $116 \cdot 25$ & $1 \cdot 44$ & $115 \cdot 52$ & 1.52 & $111 \cdot 22$ & 1.54 & 0.043 \\
\hline Model $2 \ddagger$ & 116.45 & 1.60 & 116.06 & 1.66 & 111.58 & 1.66 & 0.07 \\
\hline Model $3 \S$ & 116.54 & 1.59 & 115.96 & 1.65 & 111.58 & 1.65 & 0.067 \\
\hline
\end{tabular}

*Obtained by analysis of covariance.

tControlled for age, gender, birth date, birth order and breast-feeding pattern. ¥Further adjusted for maternal age, mother's education, father's education and parental occupation.

\$Additionally controlled for BMI.

||Mean value was significantly different from that of the first tertile.

age, gender, birth date, birth order and breast-feeding pattern $(P=0 \cdot 045)$. However, when the analysis was further adjusted for mother's age, mother's education, father's education, parental occupation and BMI, the difference between mean intelligence scores across tertiles of refined carbohydrate intake was no longer significant.
Table 3 Regression coefficients and $95 \%$ confidence intervals for the association between refined carbohydrate consumption and non-verbal intelligence scores: children $(n$ 245) aged 6-7 years, Tehran, Iran

\begin{tabular}{lrccr}
\hline & $\beta$ & $95 \% \mathrm{Cl}$ & $R^{2}$ & $P$ value \\
\hline Crude & -11.359 & $-18.897,-3.822$ & 0.039 & $<0.001$ \\
Model 1* & -9.047 & $-16.406,-1.688$ & 0.187 & 0.016 \\
Model 2† & -8.230 & $-16.286,-0.174$ & 0.230 & 0.045 \\
Model 3‡ & -8.495 & $-16.506,-0.484$ & 0.244 & 0.038 \\
\hline
\end{tabular}

*Controlled for age, gender, birth date, birth order and breast-feeding pattern. tFurther adjusted for mother's age, mother's education, father's education and parental occupation.

$\ddagger$ Additionally controlled for BMI.

Findings from linear regression analysis are provided in Table 3. The relationship between refined carbohydrate consumption and non-verbal intelligence scores was examined in a crude model as well as in additional models controlling for confounders. In the crude model, there was a significant inverse association between refined carbohydrate consumption and non-verbal intelligence scores $(\beta=-11 \cdot 359, P<0 \cdot 001)$. This relationship remained significant in multivariate analysis after controlling 
for age, gender, birth date, birth order and breast-feeding pattern $(\beta=-9 \cdot 047, P=0 \cdot 016)$. Additional adjustment for mother's age, mother's education, father's education and parental occupation did not alter the findings ( $\beta=-8 \cdot 230, P=0 \cdot 045)$. Even after further control for BMI, the inverse association of refined carbohydrate consumption with non-verbal intelligence scores remained statistically significant $(\beta=-8 \cdot 495, P=0 \cdot 038)$.

\section{Discussion}

The present cross-sectional study performed among a small group of Tehrani children found an inverse relationship between refined carbohydrate intake and non-verbal intelligence. This association remained statistically significant after adjustment for potential confounders. We found that mean scores for non-verbal intelligence decreased as refined carbohydrate consumption increased. To the best of our knowledge, our study is the first to examine the association between habitual intake of refined carbohydrates and non-verbal intelligence among children.

Few studies are available examining long-term habitual dietary intakes in relation to IQ; however, numerous investigations have explored the effect of short-term dietary intakes on cognitive performance albeit with inconsistent results ${ }^{(15)}$. For instance, a positive effect of a low-glycaemic-load breakfast on memory has been shown as compared with a high-glycaemic-load breakfast $^{(31-33)}$. Verbal IQ, like cognition and learning ability, is more affected by environment in comparison with nonverbal IQ while performance IQ is more likely to be innate $^{(25)}$. Although Raven's Progressive Matrices is a nonverbal test which measures 'fluid' rather than environmentally conditioned 'crystallized' intelligence ${ }^{(34)}$, surprisingly our results indicated that performance IQ can be related to usual refined carbohydrate consumption. Northstone et $a l .{ }^{(25)}$ found an inverse association between a 'processed' dietary pattern at age 3 years and IQ at age 8.5 years. The 'processed' dietary pattern in their study was greatly loaded with foods high in sugar and fat. The authors came to the conclusion that diet in terms of dietary patterns can affect IQ, not only verbal but also non-verbal IQ. However, they failed to find a significant relationship between the "processed' dietary pattern at age 4 or 7 years and IQ at age 8.5 years. In the current study, we were not able to calculate the fat, protein and sugar contents of foods included in the FFQ. Therefore, we cannot differentiate the effect of individual macronutrients on intelligence. However, as refined carbohydrate intake in our study is based primarily on processed grains, our findings are not so much different from those reached by Northstone et al. ${ }^{(25)}$.

Refined carbohydrate consumption has increased in recent years ${ }^{(35)}$ and has been connected to many chronic diseases. This is particularly relevant for Asian countries where items containing refined carbohydrates, namely rice and white bread, are the staple foods. Findings of the current study support the previous findings on the harmful effects of refined carbohydrate consumption on human health.

The mechanisms by which high intake of refined carbohydrates can affect non-verbal IQ in man remain to be understood. An experimental study has found that consumption of a high-fat, refined carbohydrate diet for 2 months resulted in a significant reduction in hippocampal level of brain-derived neurotrophic factor and spatial learning performance ${ }^{(36)}$. Maintaining animals on this diet for a duration of 24 months resulted in a significant decrease of synapsin I mRNA and protein (total and phosphorylated), cyclic AMP-response elementbinding protein mRNA and protein (total and phosphorylated) and growth-associated protein 43 mRNA. All of these proteins are involved in neurotransmitter release and are required for neurite outgrowth, learning and memory ${ }^{(36)}$. Furthermore, the short-term beneficial influence of carbohydrate or glucose consumption on cognitive performance has been suggested to occur via affecting serum insulin ${ }^{(37,38)}$, ghrelin $^{(39)}$ or cortisol ${ }^{(40)}$ levels. However, it is not clear if habitual dietary intakes of refined carbohydrates exert their effects on intelligence via this mechanism.

Several limitations must be considered in the interpretation of our findings. First is the cross-sectional nature of our study which prohibits inferring a casual relationship between refined carbohydrate consumption and non-verbal IQ. Second, the modified FFQ we used in the study was designed to collect data about refined carbohydrate consumption. Therefore, data on the dietary intakes of other food groups, macronutrients and particularly total energy intake were not available. However, we adjusted our statistical analyses for BMI, as a measure of total energy intake, to control for the effect of energy intake on studied relationships. It must be kept in mind that the protein and fat contents of processed grains (from which we calculated the refined carbohydrate intake) might contribute to the glycaemic index of foods and eventually to cognitive ability. Therefore, future studies exploring the independent association between refined carbohydrate intakes and cognitive ability must consider the confounding effect from dietary fats and proteins. Third, we cannot extrapolate our findings to all students of Tehran because the study participants were confined to two western regions of Tehran. However, socio-economic status was considered in sample selection enabling us to have a broad range of dietary intakes. Fourth, the small sample size in the study might prohibit us from reaching enough statistical power for the study. As studying the association of habitual intakes of refined carbohydrates with intelligence is a novel hypothesis, even findings from small samples are of value. For better understanding of the association, prospective cohort studies with larger sample sizes would be required to confirm our findings. 
In conclusion, the present study provides evidence indicating an inverse relationship between refined carbohydrate consumption and non-verbal IQ among 6-7-yearold Tehrani children. These findings support the hypothesis that long-term dietary intakes may have positive or adverse effects on the 'fluid' intelligence. More studies are needed to confirm this hypothesis.

\section{Acknowledgements}

The data collection phase of the study was funded by the National Nutrition and Food Technology Research Institute, Tehran, Iran. Financial support for drafting the manuscript came from the Food Security Research Center, Isfahan University of Medical Science, Isfahan, Iran. None of the authors had any financial or personal conflicts of interest. All authors were involved in planning the study design. A.S.A. and N.K. contributed to the conception of the study hypothesis. Intelligence testing was supervised by A.A.E. FFQ modification was supervised by A.H.R. The data analysis was performed by A.S.A. and supervised by B.R. and A.E. A.S.A. and A.E. wrote the first draft of the manuscript. All authors were involved in revisions and final approval of the manuscript. The authors would like to thank Mohammad Afkhamin and Ashkan Ariai Nejad for their assistance in data collection and analysis of the FFQ data.

\section{References}

1. Leiva Plaza $\mathrm{B}$, Inzunza Brito $\mathrm{N}$, Perez Torrejon $\mathrm{H}$ et al. (2001) The impact of malnutrition on brain development, intelligence and school work performance. Arch Latinoam Nutr 51, 64-71.

2. Brown JL \& Pollitt E (1996) Malnutrition, poverty and intellectual development. Sci Am 274, 38-43.

3. Zhao L, Yu W, Jia F et al. (2009) The influence of the nutrient supplement for children on the nutrition and health status and intelligence of children of schooling age in the countryside of children of schooling age during 2 years. Wei Sheng Yan Jiu 38, 696-699.

4. Stein AD, Wang M, DiGirolamo A et al. (2008) Nutritional supplementation in early childhood, schooling, and intellectual functioning in adulthood: a prospective study in Guatemala. Arch Pediatr Adolesc Med 162, 612-618.

5. Qian M, Wang D \& Chen Z (2000) A preliminary metaanalysis of 36 studies on impairment of intelligence development induced by iodine deficiency. Zhonghua $Y u$ Fang Yi Xue Za Zhi 34, 75-77.

6. Gonzalez HF, Malpeli A, Etchegoyen G et al. (2007) Acquisition of visuomotor abilities and intellectual quotient in children aged 4-10 years: relationship with micronutrient nutritional status. Biol Trace Elem Res 120, 92-101.

7. Pollitt E, Saco-Pollitt C, Jahari A et al. (2000) Effects of an energy and micronutrient supplement on mental development and behavior under natural conditions in undernourished children in Indonesia. Eur J Clin Nutr 54, Suppl. 2, S80-S90.

8. Christian P, Murray-Kolb LE, Khatry SK et al. (2010) Prenatal micronutrient supplementation and intellectual and motor function in early school-aged children in Nepal. JAMA 304, 2716-2723.
9. Benton D (2001) Micro-nutrient supplementation and the intelligence of children. Neurosci Biobehav Rev 25, 297-309.

10. Schoenthaler SJ, Bier ID, Young K et al. (2000) The effect of vitamin-mineral supplementation on the intelligence of American schoolchildren: a randomized, double-blind placebo-controlled trial. J Altern Complement Med 6, $19-29$.

11. Theodore RF, Thompson JMD, Waldie KE et al. (2009) Dietary patterns and intelligence in early and middle childhood. Intelligence 37, 506-513.

12. Leigh Gibson E \& Green MW (2002) Nutritional influences on cognitive function: mechanisms of susceptibility. Nutr Res Rev 15, 169-206.

13. Messier C (2004) Glucose improvement of memory: a review. Eur J Pharmacol 490, 33-57.

14. Dye L \& Blundell J (2002) Functional foods: psychological and behavioural functions. $B r J$ Nutr 88, Suppl. 2, S187-S211.

15. Gilsenan MB, de Bruin EA \& Dye L (2009) The influence of carbohydrate on cognitive performance: a critical evaluation from the perspective of glycaemic load. Br J Nutr 101, 941-949.

16. Hoyland A, Lawton CL \& Dye L (2008) Acute effects of macronutrient manipulations on cognitive test performance in healthy young adults: a systematic research review. Neurosci Biobehav Rev 32, 72-85.

17. Popkin BM \& Nielsen SJ (2003) The sweetening of the world's diet. Obes Res 11, 1325-1332.

18. Gross LS, Li L, Ford ES et al. (2004) Increased consumption of refined carbohydrates and the epidemic of type 2 diabetes in the United States: an ecologic assessment. Am J Clin Nutr 79, 774-779.

19. Liu S (2002) Intake of refined carbohydrates and whole grain foods in relation to risk of type 2 diabetes mellitus and coronary heart disease. J Am Coll Nutr 21, 298-306.

20. McKeown NM, Meigs JB, Liu S et al. (2004) Carbohydrate nutrition, insulin resistance, and the prevalence of the metabolic syndrome in the Framingham Offspring Cohort. Diabetes Care 27, 538-546.

21. Preuss HG, Fournier RD, Chieuh CC et al. (1986) Refined carbohydrates affect blood pressure and retinal vasculature in spontaneously hypertensive and Wistar-Kyoto rats. J Hypertens Suppl 4, issue 3, S459-S462.

22. Fournier RD, Chiueh CC, Kopin IJ et al. (1986) Refined carbohydrate increases blood pressure and catecholamine excretion in SHR and WKY. Am J Physiol 250, E381-E385.

23. Sherafat-Kazemzadeh $\mathrm{R}$, Egtesadi $\mathrm{S}$, Mirmiran $\mathrm{P}$ et al. (2010) Dietary patterns by reduced rank regression predicting changes in obesity indices in a cohort study: Tehran Lipid and Glucose Study. Asia Pac J Clin Nutr 19, 22-32.

24. Goldbart AD, Row BW, Kheirandish-Gozal L et al. (2006) High fat/refined carbohydrate diet enhances the susceptibility to spatial learning deficits in rats exposed to intermittent hypoxia. Brain Res 1090, 190-196.

25. Northstone K, Joinson C, Emmett P et al. (2011) Are dietary patterns in childhood associated with IQ at 8 years of age? A population-based cohort study. J Epidemiol Community Health (Epublication ahead of print version).

26. Willett W (1998) Nutritional Epidemiology. New York, Oxford: Oxford University Press.

27. Azadbakht LMP, Esmailzadeh A \& Azizi F (2005) Dairy consumption is inversely associated with the prevalence of the metabolic syndrome in Tehranian adults. Am J Clin Nutr 82, 523-530.

28. Dadkhah Piraghaj M, Amini M, Hooshyarrad A et al. (2008) Qualitative and quantitative survey of elementary students, Tehran. Iranian J Nutr Food Technol 3, 31-34. 
29. Baraheni MN (1974) Raven's progressive matrices as applied to Iranian children. Educ Psychol Meas 34, 983-988.

30. World Health Organization (2001) Global Strategy for Infant and Young Child Feeding: The Optimal Duration of Exclusive Breastfeeding. Geneva: WHO.

31. Wesnes KA, Pincock C, Richardson D et al. (2003) Breakfast reduces declines in attention and memory over the morning in schoolchildren. Appetite 41, 329-331.

32. Benton D, Maconie A \& Williams C (2007) The influence of the glycaemic load of breakfast on the behaviour of children in school. Physiol Behav 92, 717-724.

33. Ingwersen J, Defeyter MA, Kennedy DO et al. (2007) A low glycaemic index breakfast cereal preferentially prevents children's cognitive performance from declining throughout the morning. Appetite 49, 240-244.

34. Stobart G (2008) Intelligence testing: how to create a monster. In Testing Times: The Uses and Abuses of Assessment, pp. 30-56. London: Taylor \& Francis.
35. Huang D, Dhawan T, Young S et al. (2011) Fructose impairs glucose-induced hepatic triglyceride synthesis. Lipids Health Dis 10, 20.

36. Molteni R, Barnard RJ, Ying Z et al. (2002) A high-fat, refined sugar diet reduces hippocampal brain-derived neurotrophic factor, neuronal plasticity, and learning. Neuroscience 112, 803-814.

37. Park CR (2001) Cognitive effects of insulin in the central nervous system. Neurosci Biobehav Rev 25, 311-323.

38. Trejo JL, Piriz J, Llorens-Martin MV et al. (2007) Central actions of liver-derived insulin-like growth factor I underlying its pro-cognitive effects. Mol Psychiatry 12, $1118-1128$.

39. Diano S, Farr SA, Benoit SC et al. (2006) Ghrelin controls hippocampal spine synapse density and memory performance. Nat Neurosci $9,381-388$.

40. Het S, Ramlow G \& Wolf OT (2005) A meta-analytic review of the effects of acute cortisol administration on human memory. Psychoneuroendocrinology 30, 771-784. 